\title{
Elevated high-sensitivity troponin does not indicate the presence of coronary artery disease in patients presenting with supraventricular tachycardia
}

\author{
Martin Murer*, Florim Cuculi*, Stefan Toggweiler, \\ Vanessa Weberndoerfer, Mabelle Young, Richard Kobza \\ Heart Centre Lucerne, Luzerner Kantonsspital, Lucerne, Switzerland
}

\begin{abstract}
Background: Patients with supraventricular tachycardia (SVT) and patients with coronary artery disease (CAD) often present with similar symptoms (chest pain, shortness of breath), similar electrocardiographic changes and elevated high-sensitivity troponin (Tn). It is not clear whether troponin reflects critical CAD or is elevated due to other causes in patients presenting with SVT. The aim of this study was to assess the role of elevated troponin in patients presenting with SVT.

Methods: Patients undergoing radiofrequency ablation (RFA) for SVT and simultaneous coronary angiography at the Heart Centre Lucerne, Switzerland between January 2010 and October 2014 were included in this analysis. Significant CAD was defined as diameter-stenosis $\geq 75 \%$ in vessels $>2.0 \mathrm{~mm}$. The level of Tn was compared between patients with the presence or absence of CAD on coronary angiography. A Tn value of $\geq 0.014 \mu g / L$ was considered as elevated.

Results: During the study period a total of 473 patients underwent RFA for SVT. The study population consisted of 326 patients (69\%, mean age $60 \pm 12$ years) who underwent invasive coronary angiography during the same session. The prevalence of significant CAD was 14\% (45/326 patients). The highest prevalence of CAD was found in patients with atrial flutter (35\%, 18/45 patients). Tn was elevated in 83\% (10/12 patients) with significant CAD and in 47\% (26/55 patients) without CAD.

Conclusions: The prevalence of $C A D$ is low in patients with SVT, which questions the role of routine invasive coronary angiography during RFA. Tn measurement did not reliably exclude or confirm CAD in these patients. (Cardiol J 2017; 24, 6: 642-648)

Key words: coronary artery disease, prevalence of coronary artery disease, supraventricular tachycardia, high-sensitivity troponin, radiofrequency ablation
\end{abstract}

\section{Introduction}

Supraventricular tachycardias (SVT) are amongst the most common cardiac arrhythmias and are related to significant morbidity and mortality [1,2]. SVT can be paroxysmal, sometimes persistent or rarely life threatening [1]. The prevalence of paroxysmal SVT is estimated to be $3.5 \%$ at an incidence of 35 per 100,000 person years [3]. Age influences the occurrence of SVT with atrial fibrillation (AF) mainly affecting the older population. The SVT population is younger than the population with other cardiovascular diseases (CVD) [3]. Furthermore, women are twice as likely to develop a paroxysmal SVT compared to men [3].

Address for correspondence: Richard Kobza, MD, PhD, Head Heart Centre Lucerne Luzerner Kantonsspital Spitalstrasse, 6000 Luzern 16, Switzerland, e-mail: richard.kobza@luks.ch

Received: 16.12.2016

Accepted: 02.05.2017

*Contributed equally 
Symptoms of patients presenting with SVT are heterogenic including palpitations, exercise intolerance, dizziness, dyspnea, angina, pre-syncope or syncope [1] and can very often not be clearly differentiated from symptoms related to other CVD (e.g. cardiac ischemia) $[4,5]$.

Coronary artery disease (CAD) is common in the Western world and can present as stable angina or as acute coronary syndrome (ACS) [6]. Prolonged cardiac ischemia leads to myocardial injury and cardiac biomarkers are being used as a tool to diagnose and quantify myocardial damage [6]. New cardiac troponin (Tn) assays have a high sensitivity to detect myocardial injury $[7,8]$ and are now widely used in clinical practice. Increased $\mathrm{Tn}$ is not only a marker for ischemic heart disease and it is well known that several more conditions can be associated with elevated Tn: e.g. heart failure, myocarditis, dissection of the aorta and chronic renal failure [9].

Patients with SVT are often assessed in the emergency department (ED) and cardiac biomarkers are regularly measured in order to exclude acute myocardial injury requiring percutaneous or surgical revascularization. In patients with SVT, elevated Tn-levels may be observed [5] but case reports and small case series indicate that elevated $\mathrm{Tn}$ in patients with SVT does not necessarily indicate the presence of CAD [10-12].

The aim of this study was to highlight the role of elevated Tn in patients presenting with SVT and undergoing invasive coronary angiography.

\section{Methods}

This was a retrospective study performed in the Heart Centre Lucerne, Luzerner Kantonsspital, Lucerne, Switzerland. The Heart Centre Lucerne is a tertiary care center performing $>350$ radiofrequency-ablations (RFA) and 2500 coronary angiograms per year.

\section{Study populations}

The cohort consisted of patients undergoing RFA for SVT who underwent invasive coronary angiography in the same session. The procedure took place within 7 days after the presentation of the patients in our ED. Coronary angiography was performed as part of routine clinical care to exclude relevant CAD. The study period lasted from January 2010 to October 2014 .

\section{Supraventricular tachycardia}

The following categories of SVT's were treated: $\mathrm{AF}$, atrial flutter (AFL), atrioventricular nodal re-entry tachycardia (AVNRT), atrioventricular reentry tachycardia (AVRT), atrial tachycardia (AT).

\section{Troponin analysis}

In patients presenting with an acute episode of SVT in our ED plasma samples were analyzed for high-sensitivity troponin (Tn) using the Roche Cobras e601 device. An increased Tn-level was defined at a value of $\geq 0.014 \mu \mathrm{g} / \mathrm{L}$. The peak value within $24 \mathrm{~h}$ post-presentation was used for statistical analysis. Patients with point-of-care troponin measurements or troponin measurements of other hospitals were excluded.

\section{Coronary angiography findings}

Significant coronary artery stenosis was defined as a diameter-stenosis $\geq 75 \%$ in two orthogonal views in a coronary artery $\geq 2.0 \mathrm{~mm}$. SYNTAX score was calculated using the web calculator [13].

\section{Ethical considerations}

Approval was granted by the regional ethical committee (Northwestern-Switzerland). All patients provided written informed consent for electronic data acquisition and follow-up examinations.

\section{Statistical analysis}

Continuous data are presented as mean value \pm the standard deviation and categorical date are given as n (\%). Comparisons were performed with Student's t test for continuous variables in cases of normal distribution and the Fisher's exact test for categorical variables. The relationship between Tn-level and the Syntax score was evaluated by linear regression analysis. A two-sided $\mathrm{p}$ value $<0.05$ was considered statistically significant. A logistic regression analysis was performed to identify predictors of CAD. The statistics were conducted with SPSS statistical program. The diagnostic value to detect significant coronary artery stenosis concerning sensitivity and specificity was admitted with a receiver operating characteristic (ROC) curve.

\section{Results}

During the study period a total of 473 patients underwent RFA. 326 (69\%) patients underwent invasive coronary angiography to exclude CAD in the same session (Fig. 1) and these patients were included in the study population.

Baseline characteristics of the study population are shown in Table 1 . Briefly, mean age was $60 \pm 12$ years and $211(65 \%)$ patients were male. 


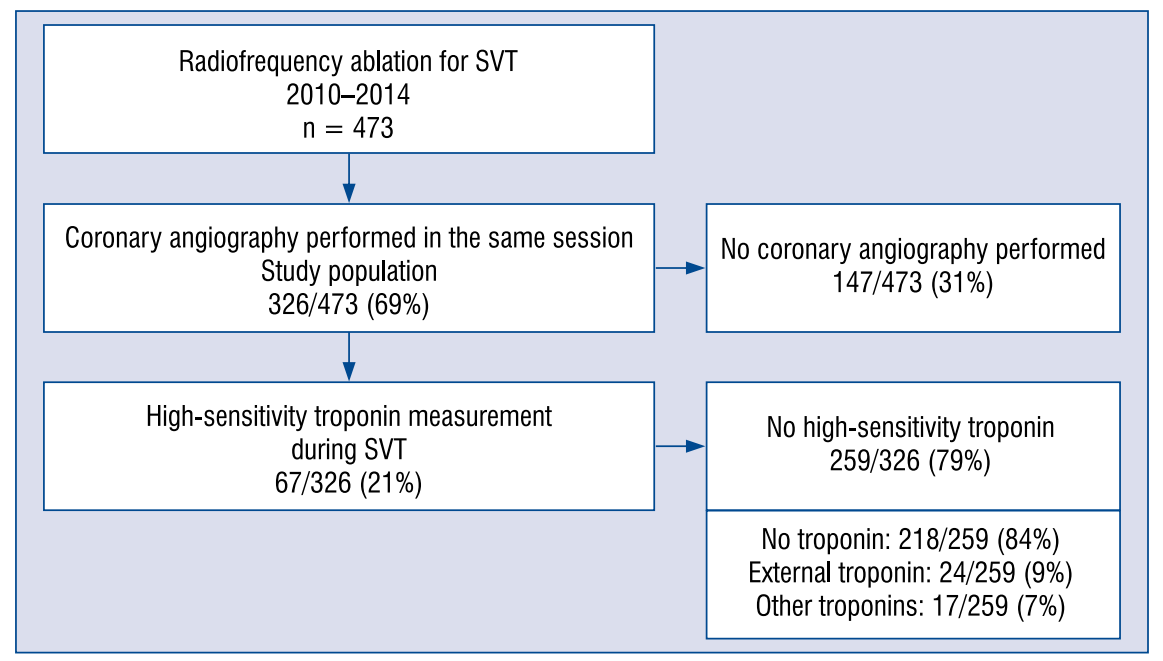

Figure 1. Definition of the study population; SVT — supraventricular tachycardia.

Table 1. Baseline characteristics of the study population $(\mathrm{n}=326)$.

\begin{tabular}{lc}
\hline Demographics & \\
Age [years] & $115(35 \%)$ \\
Sex, women & \\
Cardiovascular risk factors & $73(22 \%)$ \\
No risk factors & $141(43 \%)$ \\
Arterial hypertension & $120(37 \%)$ \\
Hypercholesterolemia & $25(8 \%)$ \\
Diabetes mellitus & $10(3 \%)$ \\
Insulin dependent & $85(26 \%)$ \\
Smoking & $70(21 \%)$ \\
Positive family history & \\
Initial symptoms & $5(2 \%)$ \\
Asymptomatic & $38(12 \%)$ \\
Typical angina & $104(32 \%)$ \\
Atypical angina & $103(32 \%)$ \\
Dyspnea & $283(87 \%)$ \\
Palpitation & $20(6 \%)$ \\
Syncope & \\
Type of SVT & $108(33 \%)$ \\
AF & $51(16 \%)$ \\
AFL & $29(9 \%)$ \\
AF + AFL & $95(29 \%)$ \\
AVNRT & $15(5 \%)$ \\
AVRT & $15(5 \%)$ \\
WPW & $10(3 \%)$ \\
\hline Ectopic AT & \\
\hline
\end{tabular}

$\mathrm{AF}$ - atrial fibrillation; AFL — atrial flutter; AVNRT — atrioventricular nodal re-entry tachycardia; AVRT — atrioventricular re-entry tachycardia; AT — atrial tachycardia; SVT — supraventricular tachycardia; WPW - Wolff-Parkinson-White syndrome
The most cardiovascular risk factor (cvRF) was arterial hypertension (43\%, 141/326 patients) and $73(22 \%)$ patients of the study population had no cvRF. Palpitation was very common $(87 \%, 283 / 326$ patients), $104(32 \%)$ of the patients had atypical angina and 38 (12\%) had typical angina. AF (33\%, 108/326 patients) and AVNRT (29\%, 95/326 patients) were the most common arrhythmias.

\section{Prevalence of CAD}

Of the 326 patients who underwent invasive coronary angiography, 45 (14\%) patients had relevant CAD and 281 (86\%) patients had normal coronaries or minor CAD (Fig. 2).

The comparison of cvRF depending on the presence or absence of significant CAD is shown in Table 2. In the univariate analysis arterial hypertension, hypercholesterolemia and male gender were all significantly more common in patients with CAD ( $\mathrm{p}<0.001$ for all).

Table 3 compares the various presenting symptoms according to the presence or absence of significant CAD. No statistically significant differences in the prevalence of symptoms were found between the groups.

The prevalence of CAD according to arrhythmia type is shown in Table 4. CAD was numerically more common in patients presenting with AFL (35\%, 18/51 patients).

\section{Role of Tn}

A total of 67 patients (14\% of the total population) presented in the ED due to tachycardia and had Tn-levels measured in the acute episode. 


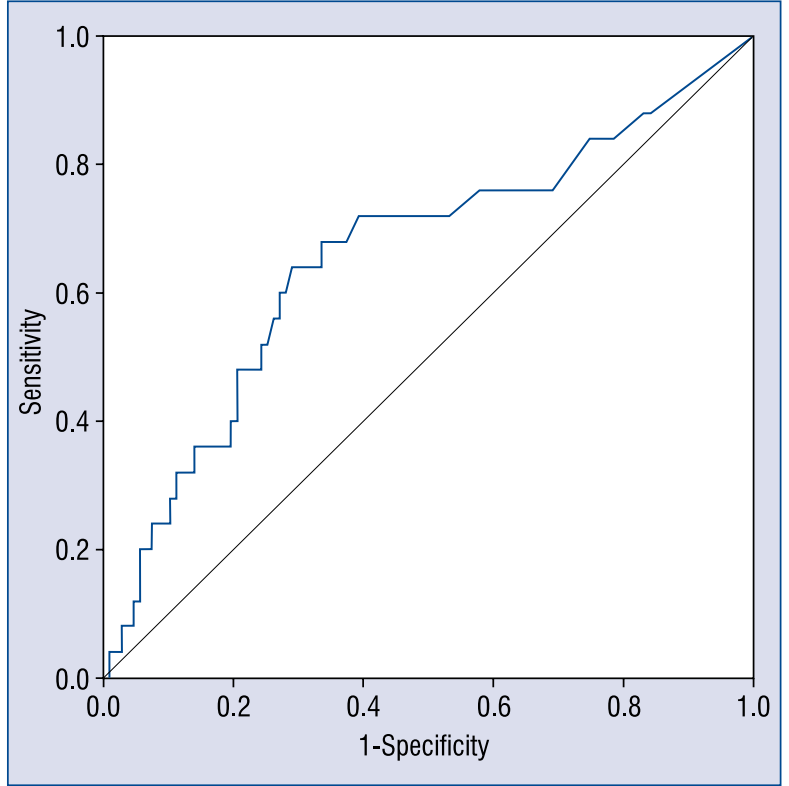

Figure 2. Receiver operating characteristic, sensitivity and specificity of high-sensitivity troponin for the diagnosis of relevant coronary artery disease in patients presenting with supraventricular tachycardia.
Cardiac troponin was elevated $(\geq 0.014 \mu \mathrm{g} / \mathrm{L})$ in $36(54 \%)$ patients, who all underwent invasive coronary angiography. Increased $\mathrm{Tn}$ was found in $83 \%$ (10/12 patients) with CAD and in $47 \%$ (26/55 patients) without CAD (Table 5). Even patients with a Tn level $>1.0 \mu \mathrm{g} / \mathrm{L}$ had normal coronaries or minor CAD and on the other hand patients with CAD and SVT had normal Tn levels. Increased Tn did not correlate with significant coronary artery stenosis and a normal cTn-level did not exclude CAD (Figs. 2, 3).

\section{Discussion}

The main findings in the present study are: a) The prevalence of CAD is low in patients presenting with SVT and undergoing RFA. b) Tn did not have a relevant impact on confirmation or exclusion of significant CAD in these patients.

Symptoms of CAD and SVT may be very similar, electrocardiogram (ECG) is often difficult to interpret regarding ischemic signs and it is common practice in many centers to perform

Table 2. Comparison of cardiovascular risk factors between patients with and without coronary artery disease (CAD) ( $n=326)$.

\begin{tabular}{lccc}
\hline & CAD $(\mathbf{n}=\mathbf{4 5} ; \mathbf{1 4} \%)$ & No CAD $(\mathbf{n}=\mathbf{2 8 1 ; 8 6 \% )}$ & P \\
\hline Age [years] & $64 \pm 8$ & $59 \pm 12$ & 0.20 \\
Male & $40(89 \%)$ & $171(61 \%)$ & $<\mathbf{0 . 0 0 1}$ \\
No risk factors & $0(0 \%)$ & $73(26 \%)$ & 0.002 \\
Arterial hypertension & $34(76 \%)$ & $107(38 \%)$ & $<\mathbf{0 . 0 0 1}$ \\
Hypercholesterolemia & $33(73 \%)$ & $87(31 \%)$ & $<\mathbf{0 . 0 0 1}$ \\
Diabetes mellitus & $8(18 \%)$ & $17(6 \%)$ & 0.74 \\
Insulin dependent & $5(11 \%)$ & $5(2 \%)$ & 0.08 \\
Nicotine & $13(29 \%)$ & $72(26 \%)$ & 0.40 \\
Positive family history & $15(33 \%)$ & $55(20 \%)$ & 0.23 \\
\hline
\end{tabular}

Table 3. Comparison of clinical characteristics between patients with and without coronary artery disease (CAD) $(n=326)$.

\begin{tabular}{lccc}
\hline & CAD $(\mathbf{n}=\mathbf{4 5}, \mathbf{1 4} \%)$ & No CAD $(\mathbf{n}=\mathbf{2 8 1 , 8 6 \% )}$ & P \\
\hline Asymptomatic & $0(0 \%)$ & $5(2 \%)$ & 1.0 \\
Typical angina & $14(31 \%)$ & $27(10 \%)$ & 0.20 \\
Atypical angina & $14(31 \%)$ & $90(32 \%)$ & 0.55 \\
Dyspnea & $21(47 \%)$ & $82(29 \%)$ & 0.06 \\
Palpitations & $37(82 \%)$ & $246(88 \%)$ & 1.0 \\
Syncope & $1(2 \%)$ & $19(7 \%)$ & 0.32 \\
\hline
\end{tabular}


Table 4. Comparison of supraventricular tachycardia between patients with and without coronary artery disease (CAD) $(n=326)$.

\begin{tabular}{lccc}
\hline & CAD $(\mathbf{n}=\mathbf{4 5}, \mathbf{1 4} \%)$ & No CAD (n = 281, 86\%) & P \\
\hline AF & $8(7 \%)$ & $100(93 \%)$ & 0.60 \\
AFL & $18(35 \%)$ & $33(65 \%)$ & 0.04 \\
AF + AFL & $3(10 \%)$ & $26(90 \%)$ & 0.21 \\
AVNRT & $14(15 \%)$ & $81(85 \%)$ & 1.0 \\
AVRT & $0(0 \%)$ & $15(100 \%)$ & 0.16 \\
WPW & $0(0 \%)$ & $15(100 \%)$ & 0.16 \\
Ectopic AT & $3(30 \%)$ & $7(70 \%)$ & 0.08 \\
\hline
\end{tabular}

AF — atrial fibrillation; AFL — atrial flutter; AVNRT — atrioventricular nodal re-entry tachycardia; AVRT — atrioventricular re-entry tachycardia; AT — atrial tachycardia; WPW - Wolff-Parkinson-White syndrome

Table 5. Comparison of high-sensitivity troponin between patients with and without coronary artery disease (CAD) $(n=67)$.

\begin{tabular}{lcccc}
\hline & Total $(\mathbf{n}=67,14 \%)$ & CAD $(\mathbf{n}=12,18 \%)$ & No CAD $(\mathbf{n}=55,82 \%)$ & P \\
\hline Troponin-hs $\geq 0.014 \mu \mathrm{g} / \mathrm{L}$ & $36(54 \%)$ & $10(83 \%)$ & $26(47 \%)$ & 0.17 \\
Troponin-hs $<0.014 \mu \mathrm{g} / \mathrm{L}$ & $31(46 \%)$ & $2(17 \%)$ & $29(53 \%)$ & 0.32 \\
\hline
\end{tabular}

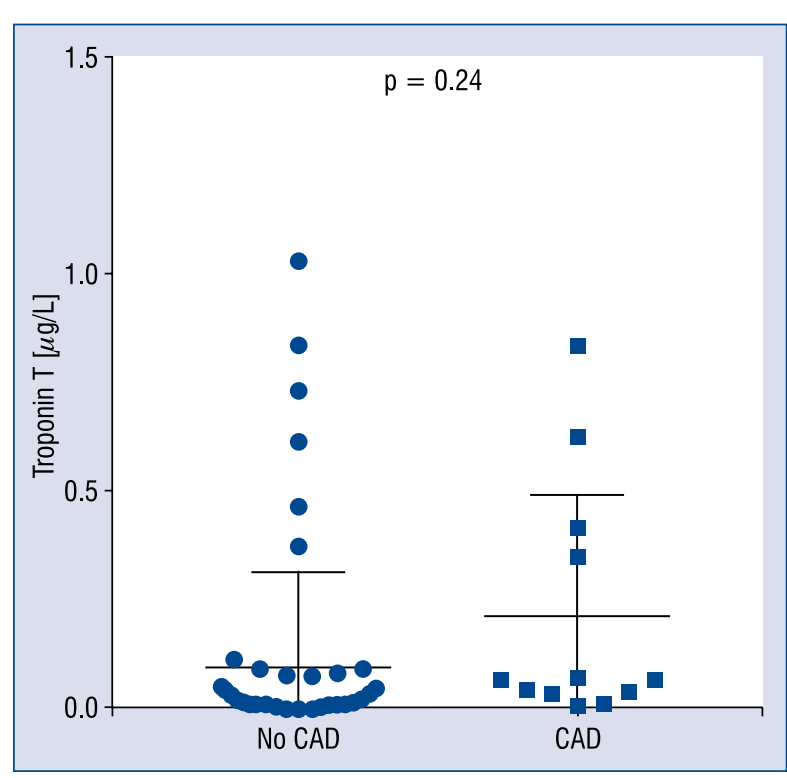

Figure 3. Comparison of high-sensitivity troponin between patients with and without coronary artery disease (CAD).

invasive coronary angiography in order to exclude or confirm CAD.

The present study demonstrates a relatively low prevalence of CAD in this group of patients and thus questions the role of routine invasive coronary angiography. This study was not able to identify a Tn cut-off, which reliably predicts the presence of CAD in patients with SVT. The assessment of $\mathrm{Tn}$ in patients with SVT, who easily convert into sinus rhythm, can lead to unnecessary diagnostic tests or wrong diagnosis [14, 15]. SalimRezaie [15] postulated that the evaluation of Tn in patients with SVT should be reserved for patients with persisting symptoms in the absence of an SVT-episode and for patients with abnormal ECG findings after conversion into sinus rhythm. On the other hand, increased Tn-levels in patients without CAD, independent of its etiology, tend to be a predictor of worse outcomes [16]. Increased Tn-levels were found to accompany an increased mortality rate [17-20] and should be rated as an indicator of critical medical status [21]. The mortality risk of patients with a ten time increased Tn-level is twice as high as for patients with normal Tn-levels [22].

There are several reasons for Tn elevations in patients with SVT. Given the fact that Tn can be elevated even in patients $<21$ years it is fair to conclude that the presence of CAD is not a condicio-sine-qua-non. From the pathophysiologic point of view heart rate, duration of SVT and mean arterial blood pressure, end-diastolic left ventricular pressure all have an important impact on coronary perfusion and could lead to mild ischemia and troponin "leak" [23]. It is also important to realize that troponin elevation does not always indicate cell death and therefore 
myocardial ischemia is only one of probably many reasons for Tn elevation [24].

Patients should be evaluated with an established risk-score to estimate the probability of $\mathrm{CAD}$, e.g. Framingham Coronary Heart Disease Risk Score [25] and the decision to perform invasive coronary angiography should be based on the risk-score rather than the presence of elevated Tn in SVT patients [26].

\section{Limitations of the study}

This study has several limitations, which need to be mentioned. It is a retrospective analysis from a single centre in Switzerland with all the limitations of retrospective analysis. The indication to perform a coronary angiography was arbitrary and made on the clinical judgement of different operators. A prospective, multi-centre study could address this important question in a more appropriate way.

\section{Conclusions}

Measurement of Tn in patients presenting with SVT was not of assistance in reliably confirming or excluding CAD. Routine invasive coronary angiography is not warranted in patients undergoing RFA and the decision should be made on clinical grounds rather than the presence of Tn elevation.

\section{Conflict of interest: None declared}

\section{References}

1. Blomström-Lundqvist C, Scheinman MM, Aliot EM, et al. ACC/ /AHA/ESC guidelines for the management of patients with supraventricular arrhythmias--executive summary: a report of the American College of Cardiology/American Heart Association Task Force on Practice Guidelines and the European Society of Cardiology Committee for Practice Guidelines (Writing Committee to Develop Guidelines for the Management of Patients With Supraventricular Arrhythmias). Circulation. 2003; 108(15): 1871-1909, doi: 10.1161/01.CIR.0000091380.04100.84, indexed in Pubmed: 14557344.

2. Camm AJ, Lip GYH, De Caterina R, et al. 2012 focused update of the ESC Guidelines for the management of atrial fibrillation: an update of the 2010 ESC Guidelines for the management of atrial fibrillation. Developed with the special contribution of the European Heart Rhythm Association. Eur Heart J. 2012; 33(21): 2719-2747, doi: 10.1093/eurheartj/ehs253, indexed in Pubmed: 22922413.

3. Orejarena LA, Vidaillet H, DeStefano F, et al. Paroxysmal supraventricular tachycardia in the general population. J Am Coll Cardiol. 1998; 31(1): 150-157, doi: 10.1016/s07351097(97)00422-1, indexed in Pubmed: 9426034.

4. Sclarovsky S, Bassevich R, Klainman E, et al. Unstable angina with tachycardia: clinical and therapeutic implications.
Am Heart J. 1988; 116(5 Pt 1): 1188-1193, doi: 10.1016/00028703(88)90438-3, indexed in Pubmed: 3189136.

5. Gassenmaier T, Buchner S, Birner C, et al. High-sensitive Troponin $\mathrm{I}$ in acute cardiac conditions: implications of baseline and sequential measurements for diagnosis of myocardial infarction. Atherosclerosis. 2012; 222(1): 116-122, doi: 10.1016/j.atherosclerosis.2012.02.007, indexed in Pubmed: 22405442.

6. Thygesen K, Alpert JS, Jaffe AS, et al. Third universal definition of myocardial infarction. Eur Heart J. 2012; 33(20): 2551-2567, doi:10.1093/eurheartj/ehs184, indexed in Pubmed: 22922414.

7. Reichlin T, Hochholzer W, Bassetti S, et al. Early Diagnosis of Myocardial Infarction with Sensitive Cardiac Troponin Assays. New England Journal of Medicine. 2009; 361(9): 858-867, doi: 10.1056/nejmoa0900428, indexed in Pubmed: 19710484.

8. Rubin Gimenez M, Twerenbold R, Jaeger C, et al. One-hour rule-in and rule-out of acute myocardial infarction using highsensitivity cardiac troponin I. Am J Med. 2015; 128(8): 861-870.e4, doi: 10.1016/j.amjmed.2015.01.046, indexed in Pubmed: 25840034.

9. Korff S, Katus HA, Giannitsis E. Differential diagnosis of elevated troponins. Heart. 2006; 92(7): 987-993, doi: 10.1136/ hrt.2005.071282, indexed in Pubmed: 16775113.

10. Miranda RC, Machado MN, Takakura IT, et al. Elevated troponin levels after prolonged supraventricular tachycardia in patient with normal coronary angiography. Cardiology. 2006; 106(1): 10-13, doi: 10.1159/000092449, indexed in Pubmed: 16601327.

11. Yeo KK, Cruz L, Hong R. Tachycardia-induced elevations in cardiac troponin in the absence of coronary artery disease. Hawaii Med J. 2006; 65(3): 86-87, indexed in Pubmed: 16724450.

12. Redfearn DP, Ratib K, Marshall HJ, et al. Supraventricular tachycardia promotes release of troponin I in patients with normal coronary arteries. Int J Cardiol. 2005; 102(3): 521-522, doi: 10.1016/j.ijcard.2004.05.076, indexed in Pubmed: 16004900.

13. Kappetein AP, Dawkins KD, Mohr FW, et al. Current percutaneous coronary intervention and coronary artery bypass grafting practices for three-vessel and left main coronary artery disease. Insights from the SYNTAX run-in phase. Eur J Cardiothorac Surg. 2006; 29(4): 486-491, doi: 10.1016/j.ejcts.2006.01.047, indexed in Pubmed: 16497510.

14. Latini R, Masson S, Anand IS, et al. Val-HeFT Investigators. Prognostic value of very low plasma concentrations of troponin $\mathrm{T}$ in patients with stable chronic heart failure. Circulation. 2007; 116(11): 1242-1249, doi: 10.1161/CIRCULATIONAHA.106.655076, indexed in Pubmed: 17698733.

15. SalimRezaie G. Do Elevated Troponins during Supraventricular Tachycardia (SVT) Predict the Presence of Coronary Artery Disease? Emergency Medicine: Open Access. 2013; 03(04), doi: 10.4172/2165-7548.1000e132.

16. Chow GV, Hirsch GA, Spragg DD, et al. Prognostic significance of cardiac troponin I levels in hospitalized patients presenting with supraventricular tachycardia. Medicine. 2010; 89(3): 141-148, doi: 10.1097/MD.0b013e3181dddb3b, indexed in Pubmed: 20453600 .

17. Fonarow G, Peacock W, Horwich T, et al. Usefulness of B-Type Natriuretic Peptide and Cardiac Troponin Levels to Predict In-Hospital Mortality from ADHERE. Am J Cardiol. 2008; 101(2): 231-237, doi: 10.1016/j.amjcard.2007.07.066, indexed in Pubmed: 18178412.

18. Henrikson CA, Howell EE, Bush DE, et al. Prognostic usefulness of marginal troponin T elevation. Am J Cardiol. 2004; 93(3): 275-279, doi:10.1016/j.amjcard.2003.10.004, indexed in Pubmed: 14759374. 
Cardiology Journal 2017, Vol. 24, No. 6

19. Horwich TB, Patel J, MacLellan WR, et al. Cardiac troponin I is associated with impaired hemodynamics, progressive left ventricular dysfunction, and increased mortality rates in advanced heart failure. Circulation. 2003; 108(7): 833-838, doi: 10.1161/01. CIR.0000084543.79097.34, indexed in Pubmed: 12912820.

20. Khan NA, Hemmelgarn BR, Tonelli M, et al. Prognostic value of troponin $\mathrm{T}$ and I among asymptomatic patients with end-stage renal disease: a meta-analysis. Circulation. 2005; 112(20): 3088-3096, doi: 10.1161/CIRCULATIONAHA.105.560128, indexed in Pubmed: 16286604.

21. Alcalai R, Planer D, Culhaoglu A, et al. Acute coronary syndrome vs nonspecific troponin elevation: clinical predictors and survival analysis. Arch Intern Med. 2007; 167(3): 276-281, doi: 10.1001/ archinte.167.3.276, indexed in Pubmed: 17296884.

22. Waxman DA, Hecht S, Schappert J, et al. A model for troponin I as a quantitative predictor of in-hospital mortality. J Am Coll Cardiol. 2006; 48(9): 1755-1762, doi: 10.1016/j.jacc.2006.05.075, indexed in Pubmed: 17084245.
23. Moore JP, Arcilla L, Wang S, et al. Characterization of Cardiac Troponin Elevation in the Setting of Pediatric Supraventricular Tachycardia. Pediatr Cardiol. 2016; 37(2): 392-398, doi: 10.1007/ s00246-015-1289-z, indexed in Pubmed: 26481117.

24. Lim C, Gaal Wv, Testa L, et al. With the "Universal Definition," Measurement of Creatine Kinase-Myocardial Band Rather Than Troponin Allows More Accurate Diagnosis of Periprocedural Necrosis and Infarction After Coronary Intervention. Journal of the American College of Cardiology. 2011; 57(6): 653-661, doi: 10.1016/j.jacc.2010.07.058, indexed in Pubmed: 21292125.

25. Lloyd-Jones D, Wilson P, Larson M, et al. Framingham risk score and prediction of lifetime risk for coronary heart disease. The American Journal of Cardiology. 2004; 94(1): 20-24, doi: 10.1016/j. amjcard.2004.03.023, indexed in Pubmed: 12963571.

26. Diez M, Talavera ML, Conde DG, et al. High-sensitivity troponin is associated with high risk clinical profile and outcome in acute heart failure. Cardiol J. 2016; 23(1): 78-83, doi: 10.5603/ CJ.a2015.0058, indexed in Pubmed: 26412605. 